\title{
The International Society of Pharmacometrics
}

\author{
Marc Pfister • Donald E. Mager • Nicholas H. G. Holford • \\ Brian Corrigan - Stacey Tannenbaum • Rene Bruno • \\ Liping Zhang · Yaning Wang · David Z. D'Argenio
}

(C) Springer Science+Business Media New York 2013

In 2011, the American Society of Pharmacometrics (ASoP) was founded and over 600 members worldwide joined ASoP within 6 months. In 2012, ASoP evolved to the International Society of Pharmacometrics (ISoP) to reflect the increasing number of international members. ISoP's growth continues and the Society currently represents over 900 members from almost 30 countries around the world. With this report, the officers and board directors of ISoP state the vision of the society, update on its activities, and invite more colleagues to join the journey for shaping the future of pharmacometrics (Fig. 1).

From ASoP to ISoP, the vision and mission of the Society remains the same, that is: to promote the central role of pharmacometrics in advancing the discovery, development, and utilization of new medicines for the treatment and prevention of disease to improve human health and well-being. The Society is guided in this effort by three overarching themes of Integration, Innovation and Impact: (i) quantitative integration of multi-source data and knowledge through the application of clinical, biomedical, biological, engineering, statistical, and mathematical concepts, made possible by (ii) continuous methodological and technological innovation, and resulting in new scientific understanding and knowledge, which in turns (iii) impacts research, discovery, development, decision making, approval, and utilization of new medicines to prevent and treat disease [1]. Consistent with this mission, ISoP (i) serves as the sponsoring organization for the

M. Pfister ( $\square)$ · D. E. Mager · N. H. G. Holford · B. Corrigan ·

S. Tannenbaum $\cdot$ R. Bruno $\cdot$ L. Zhang $\cdot$ Y. Wang ·

D. Z. D'Argenio

The International Society of Pharmacometrics (ISoP), 1200

Route 22E, Suite 2000, Bridgewater, NJ 08807, USA

e-mail: mpfister@wequantify.com
American Conference on Pharmacometrics (ACoP) and co-sponsors other International Conferences on Pharmacometrics such as the Population Approach Group of Australia \& New Zealand (PAGANZ), International Symposium in Quantitative Pharmacology (ISQP) and the World Conference on Pharmacometrics (WCoP), (ii) offers a central organization for the integration of national and international pharmacometrics communities, initiatives, consortia, and educational activities, (iii) actively partners with other scientific and medical organizations such as the American Society of Clinical Pharmacology and Therapeutics (ASCPT) and institutes such as the Critical Path Institute (C-PATH), and (iv) provides resources, mentoring, leadership opportunities, educational services, to its members, especially to its trainee members [2]. An International Advisory Group has been formed to identify how ISoP can engage more effectively with the international pharmacometric and clinical community. Through its Education Committee it provides a source for finding training programs and linking trainees with mentors.

In 2012, the Journal of Pharmacokinetics and Pharmacodynamics (JPKPD) entered into an affiliation with ISoP to accelerate the Society's efforts to advance its vision for the discipline of pharmacometrics [3]. As part of that affiliation, the ISoP Publications Committee, in coordination with JPKPD editor Dr. William Jusko, named ISoP member Dr. Peter Bonate as Associate Editor and selected five Editorial Advisory Board Members. As part of ISoP's commitment to its members, the Society will have themed issues in JPKPD each year and will solicit from its members articles throughout the year. In 2013, ISoP and JPKPD will publish a themed issue on Translational Modeling and Simulation in Neuroscience that will contain more than 10 articles. Later in the year, an ACoP 2013 Highlights issue will appear including an article on best practices in 
Fig. 1 The Journey of the International Society of Pharmacometrics (ISoP)

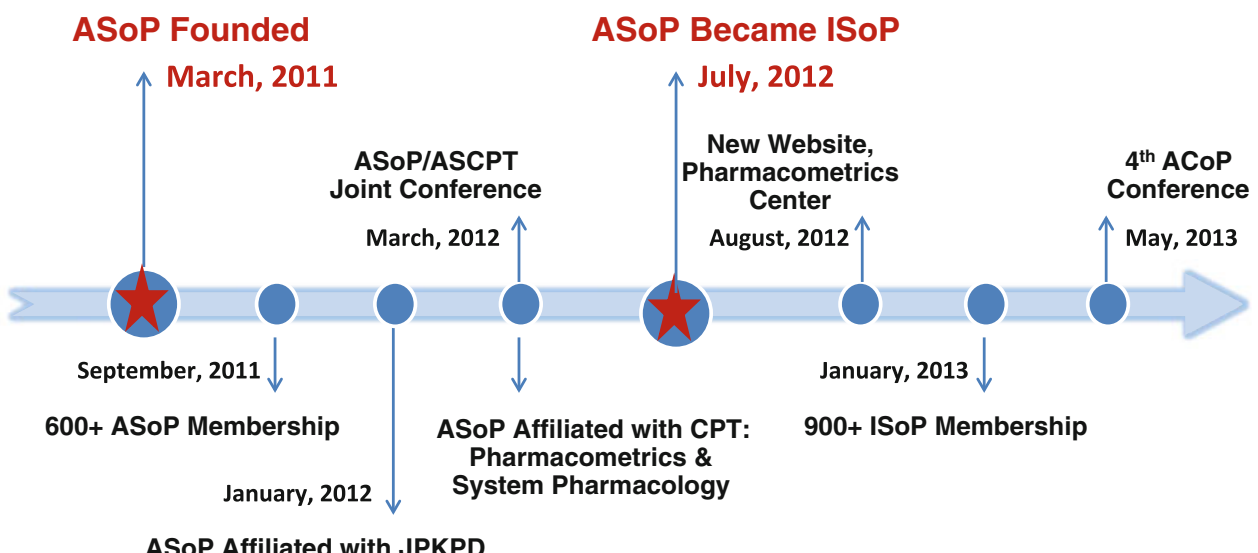

pharmacometrics. Consistent with the Society's vision and mission JPKPD is focusing on the integration, innovation and impact of pharmacometrics.

International Society of Pharmacometrics is THE international society for the science and practices of pharmacometrics. We strive to provide increasing benefits to members of ISoP, including reduced registration fees for the annual ACoP meetings and other ISoP education activities, discounted joint ASCPT/ISoP membership, and free access to JPKPD. Most significantly, membership provides you with an avenue to influence future ISoP events and activities through participation on leadership teams, so that collectively we can advance the impact of Pharmacometrics in translating scientific innovation to improving human health.

\section{References}

1. Pfister M, D'Argenio DZ (2010) The emerging scientific discipline of pharmacometrics. J Clin Pharmacol 50:6S

2. Pfister M, Brundage RC, Gastonguay MR, Miller R, Tannenbaum SJ, D'Argenio DZ (2010) Defining the future of pharmacometrics: the American Society of Pharmacometrics. J Clin Pharmacol 50:158S

3. D'Argenio DZ, Gastonguay MR, Brundage RC, Miller R, Tannenbaum SJ, Pfister M (2012) Affiliation between the American Society of Pharmacometrics and the Journal of Pharmacokinetics and Pharmacodynamics. J Pharmacokinet Pharmacodyn $39: 3$ 